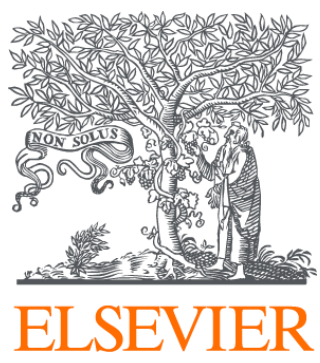

Since January 2020 Elsevier has created a COVID-19 resource centre with free information in English and Mandarin on the novel coronavirus COVID-

19. The COVID-19 resource centre is hosted on Elsevier Connect, the company's public news and information website.

Elsevier hereby grants permission to make all its COVID-19-related research that is available on the COVID-19 resource centre - including this research content - immediately available in PubMed Central and other publicly funded repositories, such as the WHO COVID database with rights for unrestricted research re-use and analyses in any form or by any means with acknowledgement of the original source. These permissions are granted for free by Elsevier for as long as the COVID-19 resource centre remains active. 


\section{Review of Imaging and Physiology Late Breaking Trials From the TCT Connect 2020 Virtual Meeting}

\section{Giorgio A. Medranda, Brian C. Case, Jason P. Wermers, Natalie Morrison, Charan Yerasi, Brian Forrestal, Chava Chezar-Azerrad, Ron Waksman *}

Section of Interventional Cardiology, MedStar Washington Hospital Center, Washington, DC, United States of America

\section{Contents}

1. Introduction. . $\cdots \cdots, 71$

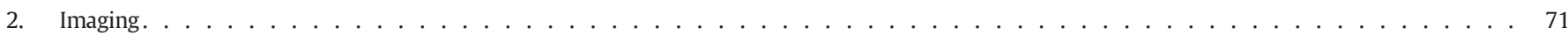

2.1. PROSPECT II: A prospective natural history study using NIRS-IVUS imaging in patients with acute myocardial infarction . . . . . . . . . . . 71

2.2. COMBINE (OCT-FFR): A prospective natural history study using OCT imaging in patients with diabetes . . . . . . . . . . . . . . . . 72

2.3. ULTIMATE: Three-year outcomes after IVUS-guided vs angiography-guided DES implantation _ . . . . . . . . . . . . 72

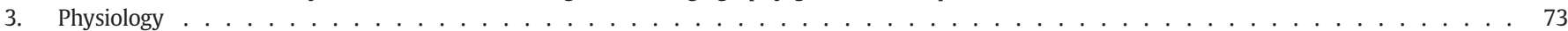

3.1. DEFINE-PCI: One-year outcomes in patients with post-PCI residual ischemia . . . . . . . . . . . . . . . . . . . . . . . 73

3.2. FORECAST: A randomized trial of CTA/FFR-CT decision-making in patients with chest pain . . . . . . . . . . . . . . . . . . . . . . . . . . . . . . 73

3.3. TARGET FFR: A randomized trial of physiology-guided PCI optimization . . . . . . . . . . . . . . . . . . . . . . . 74

3.4. DEFINE-FLOW: An observational study of deferred lesions after FFR and CFR assessment . . . . . . . . . . . . . . . . . . . . . . . . 74

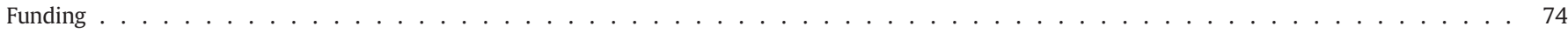

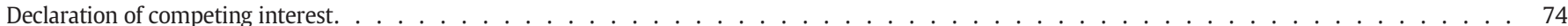

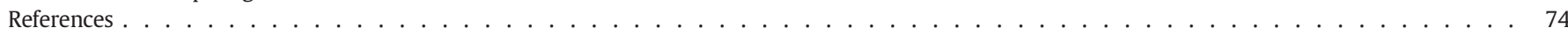

\section{Introduction}

Virtual meetings continue to replace in-person meetings across the world amidst the coronavirus disease 2019 (COVID-19) pandemic. Despite this, highly anticipated clinical research continues to be presented, with broader audiences no longer limited by distance or cost. In this article, we present a brief overview of the imaging and physiology latebreaking clinical trials presented at the Transcatheter Cardiovascular Therapeutics (TCT) Connect 2020 virtual conference that carry significant clinical implications.

\footnotetext{
* Corresponding author at: Georgetown University, Cardiovascular Research and Advanced Education, MedStar Heart and Vascular Institute, MedStar Washington Hospital Center, 110 Irving St., NW, Suite 4B-1, Washington, DC 20010, United States of America.

E-mail address: ron.waksman@medstar.net (R. Waksman).
}

\section{Imaging}

2.1. PROSPECT II: A prospective natural history study using NIRS-IVUS imaging in patients with acute myocardial infarction

\section{Presenter: Dr. David Erlinge}

Key Points: An analysis of new data from the prospective PROSPECT II natural history study of myocardial infarction (MI) patients postpercutaneous coronary intervention (PCI) suggests that near-infrared spectroscopy (NIRS) - intravascular ultrasound (IVUS) intracoronary imaging could help flag high-risk vulnerable plaques.

The findings were presented by David Erlinge, MD, of Skane University Hospital, Sweden [1]. Despite improved MI treatment options, ischemic heart disease is one of the leading causes of death globally, with acute coronary syndromes (ACS) often arising from the rupture and thrombosis of lipid-rich atherosclerotic plaques. Identifying lesions that, though currently non-obstructive, could pose future risk may enable pre-emptive strategies to stabilize the plaque and improve outcomes.

The PROSPECT II trial followed $898 \mathrm{MI}$ patients ( $83 \%$ men) with a mean age of 62.7 years (varying by 10 years) for a median of 3.7 years 


$\begin{array}{ll}\text { Abbreviations } \\ \text { ACS } & \text { acute coronary syndrome } \\ \text { AMI } & \text { acute myocardial infarction } \\ \text { CAD } & \text { coronary artery disease } \\ \text { CFR } & \text { coronary flow reserve } \\ \text { CI } & \text { confidence interval } \\ \text { COVID-19 coronavirus disease 2019 } \\ \text { CSA } & \text { cross-sectional area } \\ \text { CTCA } & \text { computed tomography coronary angiography } \\ \text { DES } & \text { drug-eluting stent } \\ \text { FFR } & \text { fractional flow reserve } \\ \text { FFR-CT } & \text { fractional flow reserve computed tomography } \\ \text { HR } & \text { hazard ratio } \\ \text { HTA } & \text { health technology assessment } \\ \text { ICA } & \text { invasive coronary angiography } \\ \text { iFR } & \text { instantaneous wave-free ratio } \\ \text { IVUS } & \text { intravascular ultrasound } \\ \text { MACCE } & \text { major adverse cardiac or cerebrovascular events } \\ \text { MACE } & \text { major adverse cardiovascular events } \\ \text { maxLCBI } & \text { 4mmaximum Lipid Core Burden Index over a 4-mm } \\ & \text { length } \\ \text { MI } & \text { myocardial infarction } \\ \text { MLA } & \text { minimal lumen area } \\ \text { NHS } & \text { National Health Service } \\ \text { NICE } & \text { National Institute for Health and Care Excellence } \\ \text { NIRS } & \text { near-infrared spectroscopy } \\ \text { NSTEMI } & \text { non-ST-segment elevation myocardial infarction } \\ \text { OCT } & \text { optical coherence tomography } \\ \text { OR } & \text { odds ratio } \\ \text { PCI } & \text { percutaneous coronary intervention } \\ \text { PIOS } & \text { physiology-guided incremental optimization strategy } \\ \text { SAQ-AF } & \text { Seattle Angina Questionnaire Angina Frequency } \\ \text { STEMI } & \text { ST-elevation myocardial infarction } \\ \text { TCFA } & \text { thin cap fibroatheroma } \\ \text { TCT } & \text { Transcatheter Cardiovascular Therapeutics } \\ \text { TLR } & \text { target lesion revascularization } \\ \text { TVMI } & \text { target vessel myocardial infarction } \\ \text { TVF } & \text { target vessel failure } \\ & \\ & \end{array}$

between 2014 and 2017 following successful PCI. Patients with troponin-positive MI (ST-elevation myocardial infarction [STEMI] > $12 \mathrm{~h}$ or non-ST-elevation myocardial infarction [NSTEMI]) were prospectively enrolled across Sweden, Denmark, and Norway, with combination NIRS-IVUS intravascular catheter imaging performed after successful PCI of all culprit lesions in the proximal 6 to $8 \mathrm{~cm}$ of all three coronary arteries. A total of 3629 untreated non-culprit lesions were characterized using NIRS-IVUS, IVUS for plaque burden and minimal lumen area (MLA), and NIRS for lipid level.

The primary and major secondary endpoints were the covariateadjusted relationship between lipid-rich plaques, defined as those with the highest quartile of the NIRS-defined maximum Lipid Core Burden Index over a 4-mm length ( $\operatorname{maxLCBI}_{4 \mathrm{~mm}}$ ) and patient-level and lesion-level major adverse cardiovascular events (MACE), respectively. The researchers also assessed the maxLCBI ${ }_{4 \mathrm{~mm}}$ cutoff of 400 or more as a secondary sensitivity analysis. IVUS-defined parameters of plaque burden of $70 \%$ or more and MLA of $4 \mathrm{~mm}^{2}$ or less were also assessed to identify high-risk vulnerable plaque. Subsequent MACE, either requiring revascularization or with rapid lesion progression, were then monitored on the basis of follow-up angiography arising from either originally treated culprit or untreated non-culprit lesions.

The researchers found that adverse events within 4 years occurred in $13.2 \%$ of patients, with the highest percentage of events (8\%) arising from untreated non-culprit lesions with a mean baseline diameter stenosis of $46.9 \%$. PROSPECT II showed that patients with a high plaque burden had a threefold increase in patient-level risk (odds ratio [OR], 3.09; 95\% confidence interval [CI]: 1.65, 5.76), and an 11-fold increase in lesion-level risk (OR, 11.37; 95\% CI: 5.60, 23.11). Those with highlipid plaques had patient risk doubled from $4.8 \%$ to $10.1 \%$, and on the lesion level a sevenfold increase, from $0.5 \%$ to $3.8 \%$.

NIRS-IVUS-identified plaques with both high lipid content and high plaque burden had a substantially higher risk, with 7\% MACE risk at the lesion level. Furthermore, the investigators found that having neither high plaque burden nor high lipid level in a plaque resulted in a very low risk of events (0.2\%). During the presentation, Erlinge said the findings should at least prompt treatment of patients found to have these kinds of "high-risk" plaques with lipid-lowering therapy and implement a preventative strategy. He and fellow researchers now call for further studies prospectively using this information to improve outcomes for high-risk patients with coronary artery disease (CAD).

The study received funding from Abbott Medical Devices, InfraReDx, and The Medicines Company.

\subsection{COMBINE (OCT-FFR): A prospective natural history study using OCT imaging in patients with diabetes}

\section{Presenter: Dr. Elvin Kedhi}

Key Points: A prospective study shows that diabetic patients with negative fractional flow reserve (FFR) $(>0.80)$ and high-risk features on optical coherence tomography (OCT)-thin cap fibroatheroma (TCFA) have higher adverse events during follow-up.

FFR is used to guide the revascularization strategy in patients with intermediate lesions. The conventional approach is that FFR-negative lesions $(>0.80)$ are treated medically and FFR-positive lesions $(\leq 0.80)$ are treated with revascularization. Elvin Kedhi, MD, $\mathrm{PhD}$, of Liberal Universiteit Brussel, Belgium, presented the results of the COMBINE study [2].

In the COMBINE (OCT-FFR) study, diabetic patients undergoing angiography with at least one non-culprit lesion with stenosis of $40 \%$ to $80 \%$ and FFR $>0.80$ underwent OCT and were assigned to a group based on TCFA identified on OCT. The primary endpoint was target lesionrelated MACE, defined as cardiac death, target vessel myocardial infarction (TVMI), clinically driven target lesion revascularization (TLR), or hospitalization due to unstable or progressive angina at 18 months.

A total of 550 patients were enrolled, 547 entered the study procedure, 423 had an FFR $>0.80$, and 112 had an FFR $\leq 0.80$. Out of 423 patients who had an FFR $>0.80,390$ had OCT. In 292 patients, there was no evidence of TCFA, and 98 patients had TCFA. At 18 months, the primary endpoint was significantly higher in the group with TCFA (13.3\% vs. 3.1\%; hazard ratio [HR], 4.7; 95\% CI: 2.0, 10.9; $\mathrm{p}=0.0004$ ). Results of the primary endpoint were driven by significantly higher rates of TVMI, TLR, and hospitalization for unstable or progressive angina. On multivariable Cox regression analysis, TCFA and minimal lumen area decrease of $1 \mathrm{~mm}^{2}$ were noted to be significant predictors of adverse events.

Kedhi concluded that in diabetic patients, more than $25 \%$ of all FFRnegative lesions have high-risk features on OCT (TCFA), and these patients have a significantly higher risk of target lesion-related adverse events at 18 months. Kedhi suggested that combined FFR and OCT can improve the lesion assessment in these high-risk patients and should be the preferred approach.

This study was funded by a research grant from St. Jude Medical (Abbott).

2.3. ULTIMATE: Three-year outcomes after IVUS-guided vs angiographyguided DES implantation

Presenter: Dr. Jun-Jie Zhang

Key Points: At 3 years, IVUS guidance for complex coronary lesions undergoing drug-eluting stent (DES) implantation continued to show a 
significantly lower target vessel failure (TVF) rate than DES implantation with angiography guidance alone.

Jun-Jie Zhang, MD, PhD, of Nanjing First Hospital, China, presented the 3-year results of the ULTIMATE trial, which showed a sustained advantage for IVUS that was first reported with the 1-year results, which Zhang presented at TCT 2018 [3]. The 3-year study results were also simultaneously published online in JACC: Cardiovascular Interventions [4].

The ULTIMATE trial enrolled 1448 all-comers with de novo lesions between August 2014 and May 2017 in 8 Chinese centers. The patients were randomized 1-to-1 to undergo IVUS or angiography guidance. IVUS-defined criteria for optimal stent deployment were the following: minimal lumen cross-sectional area (CSA) $>5.0 \mathrm{~mm}^{2}$ in the stented segment or $90 \%$ of the distal reference CSA; plaque burden $<50 \%$ at $5 \mathrm{~mm}$ proximal or distal to the stent edge; and no edge dissection involving media with length $>3 \mathrm{~mm}$.

At baseline, there were no differences between the two groups, with mean age 65.2 years in the IVUS group and 65.9 years in the angiography group, $73.9 \%$ of IVUS patients were men compared with $73.2 \%$ of angiography patients, and there were similar rates in both groups of hypertension, diabetes, current smoking, unstable angina, and acute myocardial infarction (AMI). At 1 year, the TVF rate was much lower in the IVUS group ( $2.9 \%$ vs. $5.4 \%$; HR, $0.53 ; 95 \% \mathrm{CI}: 0.31,0.90 ; \mathrm{p}=$ 0.02 ). This advantage was sustained at 3 years ( $6.6 \%$ vs. $10.7 \%$; HR, 0.60 ; $95 \%$ CI: $0.42,0.87 ; p=0.01$ ), largely driven by clinically driven target vessel revascularization (4.5\% vs. 6.9\%; HR, 0.64; 95\% CI: 0.41, 1.00; $\mathrm{p}=0.05$ ). Finally, a comparison of optimal vs. suboptimal PCI showed a significantly lower TVF rate in patients who underwent optimal PCI (4.2\% vs. 9.2\%; HR, 0.44; $95 \%$ CI: $0.24,0.81$ ).

The ULTIMATE trial was funded by the National Natural Science Foundation of China.

\section{Physiology}

3.1. DEFINE-PCI: One-year outcomes in patients with post-PCI residual ischemia

\section{Presenter: Dr. Manesh Patel}

Key Points: While an instantaneous wave-free ratio (iFR) cutoff of $\leq 0.89$ can tell clinicians when to revascularize, a different cutoff, $\geq 0.95$, might show clinicians how to optimally revascularize.

Manesh Patel, MD, of Duke Clinical Research Institute, presented the 1-year follow-up results of the DEFINE-PCI study [5]. The objectives were to assess the change in the Seattle Angina Questionnaire Angina Frequency (SAQ-AF) score, assess clinical events and perform a post hoc analysis to determine whether a post-PCI iFR value is associated with improved outcomes.

The top-line DEFINE-PCI results were reported at the American College of Cardiology (ACC) Scientific Sessions 2019. The prospective, observational study evaluated blinded iFR pullback in 500 patients who underwent PCI. The primary endpoint was the rate of residual ischemia, defined as iFR $<0.90$, after angiographically successful PCI; $24 \%$ of patients met this definition, and $81.6 \%$ of this was attributable to angiographically inapparent focal lesions. The baseline SAQ-AF score was $73.3 \pm 22.8$, and it rose by at least 10 points at 12 months in $63.1 \%$ of patients with a post-PCI iFR $<0.90$ as compared with $71 \%$ of patients with a post-PCI iFR $\geq 0.90$, a difference that did not reach statistical significance $(\mathrm{p}=0.17)$. In the most symptomatic patients (baseline SAQ-AF $\leq 60)$, SAQ-AF rose by at least 10 points more frequently in patients with a post-PCI iFR $\geq 0.95$ compared with those with a post-PCI iFR $<0.95$ (100.0\% vs. $88.5 \%$; $=0.01$ ).

Furthermore, patients with post-PCI iFR $\geq 0.95$ had a significantly lower composite clinical event rate (cardiac death, spontaneous MI, or clinically driven target vessel revascularization) at 1 year than patients with post-PCI iFR $<0.95$ ( $1.8 \%$ vs. $5.7 \%$; HR, 3.38; $95 \% \mathrm{CI}$ : 0.99, 11.6; $\log$-rank $\mathrm{p}=0.04)$. This was also true when examining only the composite of cardiac death or spontaneous myocardial infarction ( $0.0 \%$ vs. $3.2 \%$; $\log$-rank $\mathrm{p}=0.02)$. The clinical effectiveness of post-PCI iFR $\geq 0.95$ to identify and eliminate post-PCI ischemia will be studied in the prospective randomized DEFINE-GPS trial.

The DEFINE-PCI study received funding from Philips/Volcano Corp.

3.2. FORECAST: A randomized trial of CTA/FFR-CT decision-making in patients with chest pain

Presenter: Dr. Nick Curzen

Key Points: An analysis of computed tomography coronary angiography (CTCA) assessment of FFR in UK patients with stable chest pain, recruited for the FORECAST trial, showed the non-invasive procedure in FFR measurement did not significantly reduce overall cardiac medical costs within 9 months compared to routine care.

CTCA did reduce the need for invasive coronary angiography (ICA), which would be "very attractive" to patients, study lead Nick Curzen, PhD, BM (Hons), University of Southampton, said while presenting the results [6]. FFR measurement based on computed tomography (FFR-CT) is a novel, well-validated non-invasive measure to determine the presence and extent of CAD, combined with a physiological assessment of vessel-specific ischemia in patients with chest pain, the researchers note in their abstract. Previous data have suggested FFR-CT could reduce the need for invasive angiography, thereby protecting patient safety. Observational findings from the UK health technology assessment (HTA) body, the National Institute for Health and Care Excellence (NICE), the authority that issues guidance over interventions for reimbursement for the National Health Service (NHS) England and Wales, also suggest that financial savings could be made through use of CTCA and FFR-CT as a frontline strategy. NICE, therefore, already recommends FFR-CT in routine clinical practice, although the clinical effectiveness and economic impact of this compared to other tests in the evaluation of stable chest pain had not previously been tested in a randomized trial.

The FORECAST randomized trial was aimed at addressing this, laying out the economic as well as clinical impact of non-invasive cardiac tests, including FFR-CT compared with routine options such as ICA, coronary revascularization, hospitalization for a cardiac event, and cardiac medications. The primary endpoint was total cardiac medical cost through 9 months. Secondary endpoints included major adverse cardiac or cerebrovascular events (MACCE), revascularization, angina severity, and quality of life. FORECAST recruited 1400 patients in 11 UK centers with new-onset stable chest pain and a mean age of 60 years (ranging from 25 to 89 years), not including angina patients, those with a need for further testing, those with prior coronary revascularization, or those unsuitable for CTCA. The subjects were randomized 1-to-1 to receive either CTCA with FFR-CT or routine assessment under NICE guidelines, including ICA (47,6.7\%), stress echo (103, 14.7\%), nuclear perfusion scan $(13,1.9 \%)$, CTCA $(430,61.4 \%)$, exercise tolerance test (70, 10\%), stress magnetic resonance imaging $(1,0.1 \%)$, or for 36 of the patients (5.1\%), no test.

Of the 700 patients randomized to CTCA with FFR-CT, 675 (96\%) underwent CTCA, with 220 (33\%) of these patients having a coronary stenosis of $40 \%$ or more in at least one major epicardial vessel of a diameter suitable for revascularization given FFR-CT analysis. The 415 (61\%) patients with an epicardial lesion not above $40 \%$ did not receive FFR-CT, while a further 39 (6\%) could not receive this analysis because of quality or technical reasons. The researchers found that 126 of the patients undergoing FFR-CT had at least one vessel with FFR-CT of less than 0.8 . The cost in the NHS was not reduced for patients assessed by CTCA with FFRCT compared to those tested with routine care, at $£ 1605.50$ (\$2069.80) versus $£ 1491.46$ (\$1926.07), respectively. However, the researchers found that the use of ICA was $22 \%$ lower in the test group (136 patients, $19.4 \%$ ) than in the reference group, in which 175 patients (25\%) had to undergo the more invasive procedure. There were also no significant 
differences in the rates of MACCE or revascularization between the test and control groups.

The trial was sponsored by University Hospital Southampton.

\subsection{TARGET FFR: A randomized trial of physiology-guided PCI optimization}

Presenter: Dr. Damien Collison

Key Points: The number of patients found to have suboptimal FFR following PCI in the TARGET FFR trial was "surprisingly" high, according to the lead researcher, although a physiologically guided optimization strategy did improve the rates compared to standard care.

The results of the TARGET FFR trial were reported by Damien Collison, MD, of the Golden Jubilee National Hospital [7]. There is high uncertainty over the number of patients either above the favorable 0.90 threshold for FFR or left still below the 0.80 risk-level following $\mathrm{PCI}$. There are limited data for either outcome in everyday practice, as registry data suggest that the proportion of patients achieving FFR of 0.90 or more post-PCI ranges from $21 \%$ to $100 \%$. TARGET FFR, therefore, set out to assess whether a physiologically guided optimization strategy for PCI could achieve a clinically meaningful increase (by 20\%) in the proportion of patients who achieve a final post-PCI FFR of 0.90 or more compared to standard care. The secondary measure was the proportion of patients with a final post-PCI FFR result of 0.80 or less.

The researchers randomized 260 patients ( 1 to 1 ) between March 2018 and November 2019 following angiographically successful PCI for either stable angina or medically stabilized NSTEMI to receive either a physiology-guided incremental optimization strategy (PIOS) or blinded post-PCI coronary physiology measurements. All patients received a comprehensive invasive coronary physiology assessment both before and after PCI, which incorporated hyperemic and nonhyperemic indices. Patients randomized to PIOS had post-PCI physiology analyses disclosed to the operators, and FFR pullback assessments guided further protocolized optimization measures such as additional post-dilation and/or stenting.

Pre-randomization, the initial angiographically guided post-PCI FFR results were available in 238 of the 260 patients as adjudicated by the core lab. Of those, $32 \%$ had FFR of 0.90 or more. A plurality (39\%) had FFR rates between 0.81 and 0.89 , while $29 \%$ still had FFR of 0.80 or less. The rate of final post-PCI FFR of 0.90 or more, the primary endpoint, was 10 percentage points higher for the PIOS group (38.1\% vs. $28.1 \%)$, but this was not statistically significant $(p=0.099)$. However, the proportion of patients with a final FFR score of 0.80 or less was $18.6 \%$ in the PIOS group versus $29.8 \%$ in the control group, significantly lower at 11.2 percentage points $(p=0.045)$. Based on FFR pullback assessment of the stented vessel in the PIOS group, a target for further optimization was found in 60 of the 131 patients (46\%). Operators considered it appropriate to perform additional post-dilation core stenting in 40 of these 60 patients. Of those who had further intervention or optimization performed, the mean post-PCI FFR increased from 0.76 to 0.82 , with a rise in mean coronary flow reserve (CFR) from 3 to 4.

TARGET FFR did not hit its primary endpoint of achieving an FFR of 0.90 or higher post-PCI with a physiologically guided optimization strategy, but it did significantly increase the proportion of patients with a final FFR of 0.80 or less compared to standard care. Post-PCI FFR values of 0.90 or higher are linked to an improved cardiac prognosis, while the guideline-directed revascularization threshold cited in the study was 0.80 or less. Nevertheless, despite shock aired during the expert panel discussion at the press conference concerning the surprisingly high percentage of patients with suboptimal FFR levels post-PCI, Collison questioned how realistic it is to target 0.90 or more, noting wide variation in $\mathrm{PCI}$ practice.

The trial was funded by the UK's NHS National Waiting Times Centre Board endowment funds.
3.4. DEFINE-FLOW: An observational study of deferred lesions after FFR and CFR assessment

Presenter: Dr. Nils Johnson

Key Points: Operators should continue to use the FFR cutoff of $\leq 0.8$ in deciding to revascularize a lesion, even with an intact CFR $>2$.

These findings were presented by Nils Johnson, MD, MS, of McGovern Medical School at UT Health (Houston) and Memorial Hermann Hospital, Texas Medical Center [8]. The role of CFR for invasive assessment of coronary lesions currently remains unclear since FFR has become a reference standard $(\leq 0.80)$ decision for revascularization. Prior data have indicated that lesions with intact CFR $(\geq 2)$ do well, but there have not been any larger studies looking at outcomes based on simultaneous assessment by both FFR and CFR. Investigators of the DEFINE-FLOW study sought to answer this question.

This observational study included 455 patients enrolled from 12 sites in six countries. Of those enrolled, 430 patients (533 lesions) were protocol-treated and followed for 2 years. Stable coronary lesions underwent simultaneous FFR and CFR measurement in at least duplicate with central core lab review of the tracings. Treatment followed the local measurements according to a uniform protocol whereby only lesions with both FFR $\leq 0.8$ (termed FFR + ) and CFR $<2(\mathrm{CFR}+$ ) underwent PCI. All other combinations, including FFR $\leq 0.8$ but intact CFR $>2.0$ (termed CFR-), received initial medical therapy. FFR $>0.8$ was termed FFR-. The primary endpoint was MACE, a composite of allcause death, myocardial infarction, and any revascularization at 2 years with a non-inferiority margin of $10 \%$.

The study findings for MACE were $5.8 \%$ for FFR-/CFR-, $10.8 \%$ for FFR +/CFR-, $12.4 \%$ for FFR-/CFR+, and $14.4 \%$ for FFR +/CFR + (after PCI). The difference between FFR+/CFR- and FFR-/CFR- was 5.0\% (95\% CI: $-1.5 \%, 11.5 \% ; \mathrm{p}=0.065$ for non-inferiority). Therefore, the study found that vessels with abnormal FFR $\leq 0.8$ but intact CFR $\geq 2$ did not have non-inferior outcomes compared to FFR $>0.8$ and CFR $\geq 2$ when treated medically. FFR +/CFR- failed to meet noninferiority, meaning that the FFR cutoff still holds, regardless of the CFR reading. These findings are interesting, but given the observational nature of the study, they come with limitations, as subjects and physicians were unblinded in this study.

The DEFINE-FLOW trial was funded by Philips (previously Volcano Corporation).

\section{Funding}

This research did not receive any specific grant from funding agencies in the public, commercial, or not-for-profit sectors.

\section{Declaration of competing interest}

Ron Waksman - Advisory Board: Abbott Vascular, Amgen, Boston Scientific, Cardioset, Cardiovascular Systems Inc., Medtronic, Philips, Pi-Cardia Ltd.; Consultant: Abbott Vascular, Amgen, Biotronik, Boston Scientific, Cardioset, Cardiovascular Systems Inc., Medtronic, Philips, Pi-Cardia Ltd., Transmural Systems; Grant Support: AstraZeneca, Biotronik, Boston Scientific, Chiesi; Speakers Bureau: AstraZeneca, Chiesi; Investor: MedAlliance; Transmural Systems.

All other authors - None.

\section{References}

[1] Erlinge D. PROSPECT II: a prospective natural history study using NIRS-IVUS imaging in patients with acute myocardial infarction. Available from: https://www.tctconnect. $\mathrm{com} /$ late-breaking-clinical-trial-session-i-co-sponsored-by-jacc-vulnerable-plaquedetection-and-treatment/. [Accessed 19 October 2020].

[2] Kedhi E. COMBINE (OCT-FFR): a prospective natural history study using oct imaging in patients with diabetes. Available from: https://www.tctconnect.com/latebreaking-clinical-trial-session-i-co-sponsored-by-jacc-vulnerable-plaque-detectionand-treatment/. [Accessed 19 October 2020]. 
[3] Zhang J. ULTIMATE: three-year outcomes after IVUS-guided vs angiography guided DES implantation. Available from: https://www.tctconnect.com/late-breakingclinical-science-session-2-complex-pci-strategies/. [Accessed 19 October 2020].

[4] Gao X-F, Ge Z, Kong X-Q, Kan J, Han L, Lu S, et al. Three-year outcomes of the ULTIMATE trial comparing intravascular ultrasound versus angiography-guided drug-eluting stent implantation. JACC Cardiovasc Interv. 29 Oct 2020. https://doi. org/10.1016/j.jcin.2020.10.001 [E-pub ahead of print].

[5] Patel M. DEFINE-PCI: one-year outcomes in patients with post-PCI residual ischemia. Available from: https://www.tctconnect.com/late-breaking-clinical-science-session2-complex-pci-strategies/. [Accessed 19 October 2020].

[6] Curzen N. FORECAST: a randomized trial of CTA/FFRCT decision-making in patients with chest pain. Available from: https://www.tctconnect.com/late-breaking-clinical- trial-session-3-co-sponsored-by-lancet-physiology-guided-interventions/. [Accessed 19 October 2020].

[7] Collison D. TARGET FFR: a randomized trial of physiology-guided PCI optimization. Available from: https://www.tctconnect.com/late-breaking-clinical-trial-session-3co-sponsored-by-lancet-physiology-guided-interventions/. [Accessed 19 October 2020].

[8] Johnson N. DEFINE-FLOW: an observational study of deferred lesions after FFR and CFR assessment. Available from: https://www.tctconnect.com/late-breaking-clinicaltrial-session-3-co-sponsored-by-lancet-physiology-guided-interventions/. [Accessed 19 October 2020]. 